non-anthracycline-based adjuvant chemotherapy only in patients with HER2-positive disease.

Original article Gennari A et al. (2008) HER2 status and efficacy of adjuvant anthracyclines in early breast cancer: a pooled analysis of randomized trials. J Natl Cancer Inst 100: 14-20

\section{Letrozole is superior to tamoxifen for endocrine- responsive breast cancer}

The Breast International Group (BIG) 1-98 trial has previously found that in postmenopausal women with endocrine-responsive breast cancer, the aromatase inhibitor letrozole markedly improves disease-free survival (DFS) compared with tamoxifen. Now, the BIG 1-98 investigators report that letrozole is superior to tamoxifen for the treatment of ERBB2-positive tumors.

The analysis included 3,533 women with estrogen-receptor-positive tumors, who received 5 years of monotherapy with either letrozole or tamoxifen. Receptor expression was measured by immunohistochemistry, and ERBB2 positivity was confirmed by fluorescence in situ hybridization. DFS was worse in the 239 women with ERBB2-positive tumors than in those with ERBB2-negative tumors (hazard ratio [HR] 2.09, 95\% Cl 1.59-2.76; $P<0.0001)$. The estimated 4-year DFS was $75 \%$ for the group with ERBB2-positive tumors versus $88 \%$ for those with ERBB2-negative disease. Compared with tamoxifen, letrozole improved DFS irrespective of ERBB2 status (HR 0.62, 95\% Cl 0.37-1.03 for ERBB2-positive tumors; HR 0.72, 95\% Cl 0.59-0.87 for ERBB2-negative tumors). The superiority of letrozole over tamoxifen was not influenced by progesterone-receptor status, either in patients with ERBB2-positive or in those with ERBB2-negative disease.

The authors conclude that letrozole provides a treatment benefit over tamoxifen in postmenopausal women with endocrine-responsive breast cancer, regardless of ERBB2 or progesteronereceptor status.

Original article Rasmussen BB et al. (2008) Adjuvant letrozole versus tamoxifen according to centrally-assessed ERBB2 status for postmenopausal women with endocrineresponsive early breast cancer: supplementary results from the BIG 1-98 randomised trial. Lancet Oncol 9: 23-28

\section{Lymphocyte-predominant Hodgkin's Iymphoma: patient characteristics and outcomes}

Lymphocyte-predominant Hodgkin's lymphoma ( $\mathrm{LPHL}$ ) is a rare disease, which differs substantially from classical Hodgkin's lymphoma (cHL) with regard to histopathology and clinical course. Patients with LPHL are usually treated with the protocols used for $\mathrm{CHL}$; however, the treatment of early-stage LPHL and pediatric LPHL patients is less defined. A study by Nogová et al. has compared prognosis, disease characteristics and outcomes between patients with LPHL and those with cHL.

This retrospective study included 8,298 patients previously treated within the German Hodgkin Study Group trials HD4 to HD12. Of these patients, 7,904 were diagnosed with $\mathrm{cHL}$ and 394 with LPHL. The rates of complete and unconfirmed complete remission in patients with early favorable, early unfavorable and advanced LPHL were $91.6 \%, 85.7 \%$ and $76.8 \%$, respectively. The corresponding remission rates in patients with $\mathrm{CHL}$ were $85.9 \%, 83.3 \%$ and $77.8 \%$. The overall survival rates for $\mathrm{LPHL}$ and $\mathrm{CHL}$ patients were $96 \%$ and $92 \%$, respectively $(P=0.0166)$, while the overall rates of freedom from treatment failure at a median observation time of 50 months were $88 \%$ and $82 \%(P=0.0093)$. For patients with LPHL, risk factor analysis identified advanced-stage disease $(P=0.0092)$, hemoglobin level less than $10.5 \mathrm{~g} / \mathrm{dl}(155 \mathrm{~g} / \mathrm{l}$; $P=0.0171)$, and lymphopenia $(P=0.010)$ as negative prognostic factors for freedom from treatment failure. Negative prognostic factors for overall survival included hemoglobin level less than $10.5 \mathrm{~g} / \mathrm{dl}(P=0.0014)$, age of at least 45 years $(P=0.0125)$, and advanced-stage disease $(P=0.0153)$.

These results show that $\mathrm{LPHL}$ and $\mathrm{CHL}$ differ in prognosis, disease characteristics and treatment outcomes, suggesting that different treatment strategies could be used for patients with early-stage LPHL.

Original article Nogová L et al. (2008) Lymphocytepredominant and classical Hodgkin's lymphoma: a comprehensive analysis from the German Hodgkin Study Group. J Clin Oncol 26: 434-439 